\title{
Stroke in a Patient with Recurrent Meningeal Tuberculosis and HIV Coinfection
}

\author{
Authors: \\ *Dilia Fontalvo-Rivera, ${ }^{1}$ Gustavo Mora-García, ${ }^{1}$ Gustavo Jiménez- \\ Borré, ${ }^{2}$ Javier Segovia-Fuentes, ${ }^{1}$ Doris Gómez-Camargo' \\ 1. Universidad de Cartagena, Colombia \\ 2. Centro Médico Crecer, Cartagena, Colombia \\ *Correspondence to diliafontalvor@gmail.com \\ Disclosure: \\ The authors have declared no conflicts of interest. \\ Received: \\ 26.03.21 \\ Accepted: \\ 23.08.21 \\ Keywords: \\ Cerebrovascular accident (CVA), HIV, meningitis, stroke, tuberculosis (TB). \\ Citation: \\ EMJ Respir. 2021;9[1]:92-97.
}

\section{Abstract}

Recurrent tuberculous meningitis can have a non-specific clinical presentation when it presents as a co-infection with HIV and can resemble other causes of subacute and chronic meningitis, leading to unwanted outcomes; for this reason, timely diagnoses are required. The most widely used microbiological diagnostic methods can be of low sensitivity or have delayed results.

The aim of this article is to present a clinical case of recurrent tuberculous meningitis with a clinical presentation of stroke due to a basal cistern ischaemic lesion in a patient co-infected with HIV, who had a microbiological confirmation and a drug sensitivity study with phenotypic and molecular tests.

\section{INTRODUCTION}

The World Health Organization (WHO) has reported that an estimated 10.0 million people (range: 8.9-11.0 million) became ill with tuberculosis (TB) in 2019. There were an estimated 1.2 million TB deaths (range: 1.1-1.3 million) among people who were HIV-negative in 2019 and an additional 208,000 deaths (range: 177,000-242,000) among people who were HIV-positive.' This group of patients have a high risk of developing TB that is directly related to the degree of immunosuppression. The spread of TB that leads to extrapulmonary TB may be present in $66 \%$ of cases, with TB in the central nervous system being the most serious type..$^{1-3}$ In particular, meningeal TB can result in significant sequelae and is the cause of death in $30-50 \%$ of patients co-infected with $\mathrm{HIV}^{3}$
It has been observed that patients who have high or normal CD4 counts have manifestations that are typical of tuberculous meningitis (MTB), whereas those patients with low CD4 cell counts tend to have subtle and non-specific clinical manifestations. Specifically, these patients exhibit presentations with later onsets and present with severe forms of the disease, such as cerebrovascular accidents (CVA) that can be haemorrhagic or ischaemic. ${ }^{4-6}$ Likewise, it has been described that the majority of cerebral infarcts that are associated with MTB are multiple, bilateral, symmetrical, and are located in the basal ganglia, the anterior thalamus, or the forelimb or knee areas of the internal capsule., ${ }^{78}$

TB is the major cause of recurrent meningitis (40-60\%). ${ }^{9}$ According to the current criteria, recurrent meningitis is defined as that type which appears within 3-4 weeks after the end 
of treatment or when a second episode occurs due to a different micro-organism after complete remission. ${ }^{10}$ Recurrent meningitis is more common in patients with immunosuppression, chronic renal failure, cystic fibrosis, head trauma, congenital malformations, and TB. ${ }^{11,12}$ Neuropathic manifestations are due to inflammatory deposits that are generated in the cerebrospinal fluid (CSF), which are located around the brainstem, cranial nerves, and the temporal and frontal lobes. ${ }^{10,13}$

The diagnosis of MTB is based on the findings of clinical examinations, imaging, and laboratory studies. CSF studies include Ziehl-Neelsen (ZN) staining, cultures for Mycobacterium tuberculosis, and molecular biology tests. ${ }^{14-16}$

Herein, the authors present a clinical case of recurrent MTB, with a clinical presentation of stroke due to infarction in the basal cisterns in a patient co-infected with HIV. The patient had a microbiological confirmation and a drug sensitivity study with phenotypic and molecular tests.

\section{CASE REPORT}

A 40-year-old male patient, with a history of HIV infection in the AIDS phase, was receiving treatment with bictegravir, emtricitabine, tenofovir disoproxil, and cobicistat. Upon admission, he complained of a global progressive headache, decreased visual acuity, and 3-week retrograde partial amnesia. He presented with allopsychic disorientation, visual and auditory hallucinations, and decreased co-ordination when standing and moving. His case evolved with an increase in the severity of his symptoms.

On the date of the consultation, he presented with a continuous fever; in addition, he presented with tonic-clonic movements. He also presented with weight loss, losing $10 \mathrm{~kg}$ in a 3 -month period ( $18 \%$ of his weight). At the time of physical examination: tachypnoea (respiratory rate: 55 breaths/min), tachycardia (heart rate: 110 beats/min), fever (temperature: $38{ }^{\circ} \mathrm{C}$ ), and normal blood pressure were found, with sulfur dioxide at $95 \%$ under oxygen by the tracheostomy tent. His weight was $44 \mathrm{~kg}$ (BMI: $15.2 \mathrm{~kg} / \mathrm{m}^{2}$ ), which classified him as having Grade III malnutrition. He presented with drowsiness (with a Glasgow coma score of $8 / 15$ ), cranial nerve involvement (mydriasis and limitation in external eye movement in the right eye), stiff neck, hemiparesis, hypotonia and hypoesthesia in the right hemibody, and continuous tonic movements initiated in the right upper extremity with secondary generalisation were found.

Twelve months prior to his admission, a diagnosis of bacterial meningitis was made, due to the clinical and cytochemical characteristics of the CSF, although the isolation of the causative agent was not achieved. Two weeks prior to the current admission, he had a second case of meningitis, where M. tuberculosis was isolated as the aetiological agent.

Laboratory studies upon admission showed normal blood counts, serum electrolytes, coagulation, and for liver and kidney tests. The serology results for hepatitis $B$ virus and Treponema pallidum were negative. The study of orotracheal secretions did not show positivity in the cultures for aerobes and anaerobes, and the ZN stain was negative. The viral load for HIV during the hospital stay was <40 copies/ $\mathrm{mL}$, and the CD4 count was $314 / \mathrm{mm}^{3}$. A clear CSF was obtained with a cytochemical analysis that was compatible with hypoglycorrhachia (45 g/dL with glycaemia at $92 \mathrm{~g} / \mathrm{dL}$ ), protein values of $95 \mathrm{mg} / \mathrm{dL}$ and pleocytosis (polymorphonuclear: 64\%; lymphocytes: $36 \%$ ), and adenosine deaminase (ADA) at 9 $\mathrm{IU} / \mathrm{L}$, with negative $\mathrm{KOH}$, India ink, and ZN stains (Table 1). Serological tests for Herpes I and II, as well as for John Cunningham virus, were negative. PCR results for Streptococcus pneumoniae, Haemophilus influenzae, and Neisseria meningitidis were negative in the CSF. The initial chest radiograph was normal. In both simple and contrasted brain CTs, communicating hydrocephalus was found, with discrete transependymal CSF resorption. A left basal ganglion atrophic injury was observed, as well as sequelae indicative of a vascular event. Secular images were bilaterally observed in the thalamus, without enhancement. Due to a suspicion of fungal invasion in the digestive tract, endoscopy of the upper digestive tract was performed, where changes that were suggestive of oesophageal candidiasis were observed. 
Table 1: Laboratory results of the clinical case.

\section{CSF at admission}

Clear appearance

Glucose: 45 g/dL (glycaemia: $92 \mathrm{~g} / \mathrm{dL}$ )

Proteins: $95 \mathrm{mg} / \mathrm{dL}$

Cells: $150 / \mu \mathrm{L}$

$64 \%$ PMN

Lymphocytes: 36\%

ADA: 9 IU/L (reference: $<7$ IU/L)

$\mathrm{KOH},{ }^{*}$ India ink, and Ziehl-Neelsen stains were negative

PCR for Streptococcus pneumoniae, Haemophilus influenzae, and Neisseria meningitidis were negative

\section{Second CSF}

Clear appearance

Glucose: $38 \mathrm{~g} / \mathrm{dL}$ (glycaemia: $88 \mathrm{~g} / \mathrm{dL}$ )

Cells: $315 / \mu \mathrm{L}$

Proteins: $851 \mathrm{mg} / \mathrm{dL}$

PMN: $66 \%$

Lymphocytes: 34\%

ADA: 17 IU/L (reference: $<7$ IU/L)

$\mathrm{KOH}$, India ink, and Ziehl-Neelsen results stains were negative

Positive CSF culture for Mycobacterium tuberculosis; negative for other micro-organisms

${ }^{*}$ Direct examination with $\mathrm{KOH}$ for fungal structures.

ADA: adenosine deaminase; CSF: cerebrospinal fluid; $\mathrm{KOH}$ : potassium hydroxide; PMN: polymorphonuclear leukocytes.

The patient was managed with supplemental oxygen via a tracheostomy tent in combination with isonatraemic intravenous fluids, nutritional support, phenytoin (125 $\mathrm{mg} / 8$ hours), fluconazole (200 mg/day), levetiracetam (500 mg/8 hours), and acyclovir (500 mg/8 hours).

Eleven days after admission to the intensive care unit, the patient presented with greater respiratory and neurological deterioration; thus, mechanical ventilation was required. The control chest radiograph showed an image of pulmonary consolidation in the paracardiac area, with basal interstitial infiltrates in the right lung region. Finally, treatment with cefepime (2 $\mathrm{g} / 8$ hours, intravenously) was indicated.
Three days later, the patient showed neurological, respiratory, and haemodynamic deterioration. Maintenance adjustments of metabolic, electrolyte, and mechanical ventilation parameters were performed. Brain MRI scans revealed an intense left basal ganglia lacunar infarction in the simple sequence (Figure 1A); for the post-contrast measurement, the meningeal enhancement in the peritoneal cisterns was compatible with meningitis, predominantly in the cisternae of the base, which was suggestive of a TB origin (Figure 1B). Based on a previous report, CVA was considered secondary to MTB. 


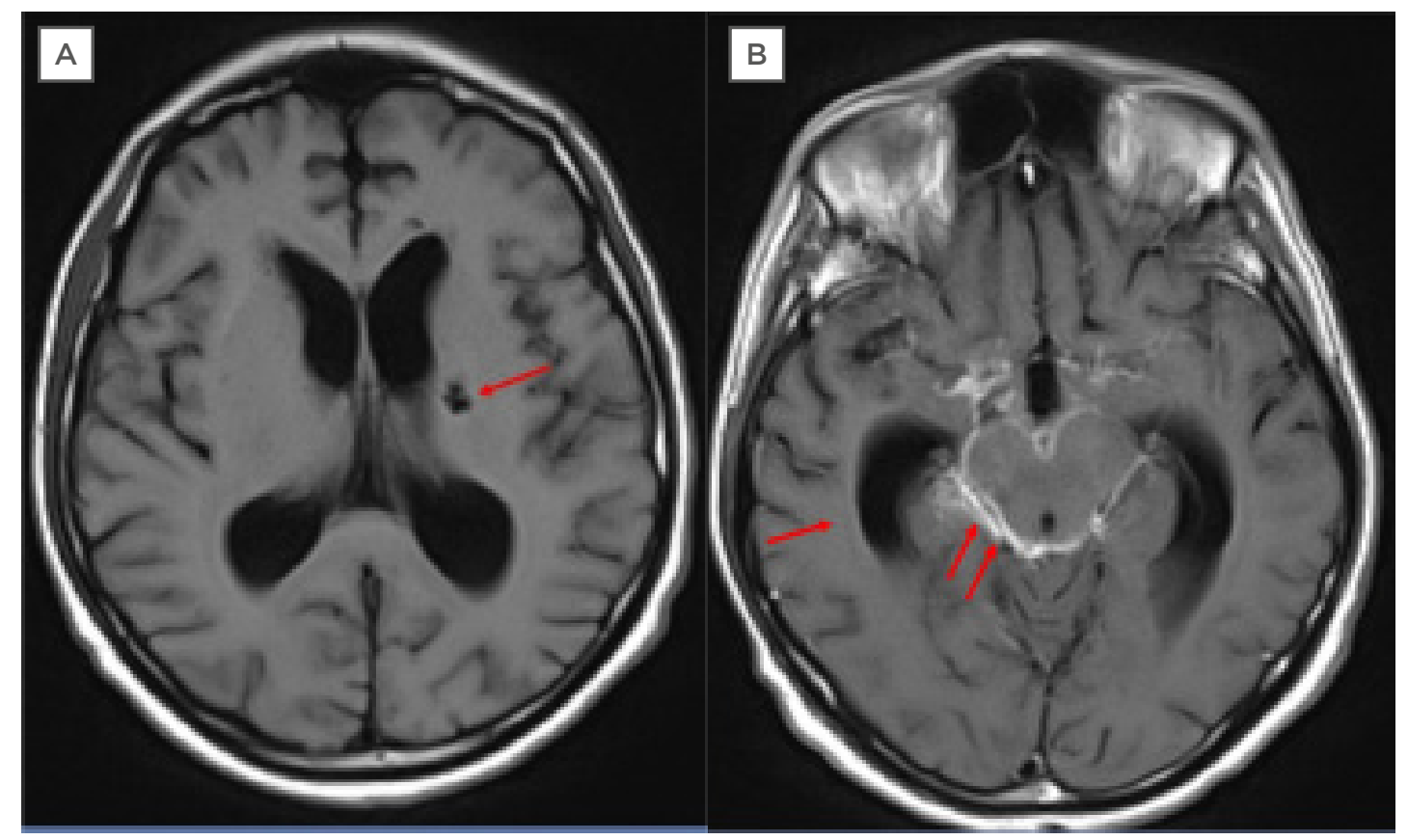

Figure 1: In meningeal tuberculosis, exudates in the basal cisterns may not be detected in simple T1 sequences. Contrast increases the signal that is usually observed in these cisterns, as occurred in this case.

A) Axial T1 sequence. Left ganglionic infarction (arrow). B) Post-gadolinium T1 sequence axial section. Dilation of the ventricular system by communication hydrocephalus (arrow). High signal of the perimesencephalic cisterns, suprasellar due to meningeal enhancement (double arrow).

A second CSF study showed an increase in the protein and ADA levels, negative ZN (Table 1). and endpoint PCR results for the insertion fragment 6110 positive for M. tuberculosis. Two weeks later, mycobacteria in the first CSF sample were grown by using an Ogawa Kudoh culture medium.

Management with dexamethasone $(16 \mathrm{mg} /$ 12 hours) and a fixed-dose anti-tuberculous therapy combined with rifampicin (450 mg/day), isoniazid (225 mg/day), pyrazinamide (1,200 $\mathrm{mg} /$ day), and ethambutol (825 mg/day) were initiated. The patient evolved towards instability without a response to new metabolic, respiratory, and therapeutic adjustments. After 33 days of his stay in the intensive care unit, he died.

\section{DISCUSSION}

The patient in the reported clinical case had risk factors for developing extrapulmonary TB, which included HIV infection, a CD4 T lymphocyte count $<200 / \mathrm{mm}^{3}$, and a state of severe malnutrition. The insidious clinical manifestations and microbiological characteristics of
M. tuberculosis may have contributed to the late confirmatory diagnosis of the disease. In this patient, an initial request for a $\mathrm{ZN}$ stain was made, but it was negative because this test requires a minimum number of bacteria to be detected, and patients with HIV infections are usually paucibacillary (the presence of $<10$ bacteria in the sample). Manifestations, including the predominance of cranial nerve involvement, compromising of the state of consciousness (with alterations in the development of thought), and deficits in muscular strength suggested a complicated neuroinfection.

Patients with HIV may have a miscellaneous chance of harbouring micro-organisms that may cause infectious meningitis. The patient described in this report presented with previous episodes of meningitis due to M. tuberculosis, which was attributed to the dissemination of pulmonary TB by reactivation. The CSF showed lymphocytic pleocytosis with increased protein and decreased glucose levels (which may be present in meningitis due to viruses), grampositive and gram-negative micro-organisms, and mycobacteria. Molecular tests were negative 
in the different biological samples except for M. tuberculosis.

Within the diagnostic standards of $T B$ is the determination of clinical, radiological, and epidemiological criteria, as well as a tuberculin skin test and a direct sputum examination, which detects only $50-80 \%$ of TB cases. Due to the increases in incident cases and TB resistance, detections with faster and less complex methods are necessary in diagnosing the disease. $16,17 \mathrm{PCR}$ is capable of demonstrating the presence of mycobacterial DNA fragments in the biological samples of patients with a clinical suspicion of $\mathrm{TB}$, as well as in samples with a negative result to ZN stains or in culture, which is particularly useful in infections of paucibacillary patients. ${ }^{18}$

Studies have shown that the genetic diversity of M. tuberculosis can have important clinical consequences. $^{19}$ Epidemiological studies have found that some genotypes of $M$. tuberculosis may be associated with extrapulmonary TB, such as polymorphisms in the mycobacterial gene $P C I D$ (phospholipase $\mathrm{C}$ ) in strains of the Beijing genotype; however, this association has not occurred in all of the studied patients. ${ }^{7}$

There are studies that have linked radiological involvement with the mycobacterial genotype. ${ }^{19}$ Lung consolidation images have been observed more frequently in the Euro-American genotype. ${ }^{20}$ Additionally, caverns and fibrosis have been more closely related to the East Asian/Beijing lineage. ${ }^{19,20}$ In this clinical case, a radiological image of pulmonary consolidation and findings in neuroimaging studies of neurological dissemination were found, with these findings due to the presence of left basal ganglia lacunar infarctions and exudates in peribronchial cisterns, which can lead to complications and death. Despite the medical history of MTB, the patient was not treated empirically with antituberculosis drugs because initial laboratory studies did not confirm the presence of M. tuberculosis. Poor adherence to treatment, co-morbidity with $\mathrm{HIV}$, and difficulty in early diagnosis may have influenced the outcome of the patient.

\section{CONCLUSION}

The patient in the clinical case had a history of HIV infection. The patient presented with three episodes of meningitis with a confirmed aetiological finding of $M$. tuberculosis on two occasions and CVA manifestations. The aetiological demonstration was potentially late, and support from the molecular biology tests helped to clarify the cause. MTB can occur in pathologies that are considered to be comorbid in TB, which includes HIV infection, thus becoming a risk factor for developing stroke. This condition can depend on viraemic conditions and CD4 counts for clinical characterisation and severity. In patients with high viral loads and low CD4 counts, an atypical clinical picture may present, which deviates the timely diagnosis of the disease and can lead to fatal outcomes or significant sequelae.

\section{References}

1. World Health Organization (WHO). Global tuberculosis report 2020. 2020. Available at: https:// www.who.int/publications/i/ item/9789240013131. Last accessed: 10 November 2020.

2. Antonucci $\mathrm{G}$ et al. Risk factors for tuberculosis in HIV-infected persons. A prospective cohort study. The Gruppo Italiano di Studio Tubercolosi e AIDS (GISTA). JAMA. 1995;274(2):143-8.

3. Garg RK, Sinha MK. Tuberculous meningitis in patients infected with human immunodeficiency virus. J Neurol. 2011;258(1):3-13.

4. Croda MG et al. Tuberculous meningitis in HIV-infected patients in Brazil: clinical and laboratory characteristics and factors associated with mortality. Int J Infect Dis. 2010;14(7):e586-91.

5. Jones BE et al. Relationship of the manifestations of tuberculosis to CD4 cell counts in patients with human immunodeficiency virus infection. Am Rev Respir Dis. 1993;148(5):1292-7.

6. Mouna E et al. Tuberculous meningitis in patients living with HIV. J Neurol Neurosur. 2016;4(1):131.

7. Misra UK et al. Stroke in tuberculous meningitis. J Neurol Sci. 2011;303(12):22-30.

8. Lammie GA et al. Tuberculous cerebrovascular disease: a review. J Infection. 2009;59(3):156-66.

9. Hildebrand J, Aoun M. Chronic meningitis: still a diagnostic challenge. J Neurol. 2003;250(6):653-60.

10. Adriani KS et al. Communityacquired recurrent bacterial meningitis in adults. Clin Infect Dis. 2007;45(5):e46-51.

11. Davis LE. Acute and recurrent viral meningitis. Curr Treat Options Neurol. 2008;10(3):168-77.

12. Tebruegge M, Curtis N. Epidemiology, etiology, pathogenesis, and diagnosis of recurrent bacterial meningitis. Clin Microbiol Rev. 2008;21(3):519-37. 
13. Garg RK. Tuberculous meningitis. Acta Neurol Scand. 2010; 122(2):75-90.

14. Vinnard C, Macgregor RR. Tuberculous meningitis in HIVinfected individuals. Curr HIV/AIDS Rep. 2009;6(3):139-45.

15. Seifert M et al. Genetic mutations associated with isoniazid resistance in Mycobacterium tuberculosis: a systematic review. PLoS One. 2015;10(3):e0119628.

16. Gazi MA et al. General and advanced diagnostic tools to detect Mycobacterium tuberculosis and their drug susceptibility: a review. Eur J Clin Microbiol. 2015;34(5):851-61.

17. da Silva RM et al. Diagnosis of the pulmonary tuberculosis by polymerase chain reaction: a comparative study between HIVpositive and -negative individuals. Braz J Microbiol. 2012;43(1):261-5.

18. Akkaya O, Kurtoglu MG. Comparison of conventional and molecular methods used for diagnosis of
Mycobacterium tuberculosis in clinical samples. Clin Lab. 2019;65(10):10-77.

19. Gagneux S, Small PM. Global phylogeography of Mycobacterium tuberculosis and implications for tuberculosis product development. Lancet Infect Dis. 2007;7(5):328-37.

20. Thwaites $\mathrm{G}$ et al. Relationship between Mycobacterium tuberculosis genotype and the clinical phenotype of pulmonary and meningeal tuberculosis. J Clin Microbiol. 2008:46(4):1363-8. 